\title{
Anonymous yes-no voting with abstention and multiple levels of approval
}

\author{
Josep Freixas, Department of Applied Mathematics 3, Technical University of Catalonia, Av. Bases de \\ Manresa, 61-73, 08242 Manresa, Spain
}

William S. Zwicker Department of Mathematics, Union College, Schenectady, NY 12308, USA

\begin{abstract}
Symmetric $(3,2)$ simple games serve as models for anonymous voting systems in which each voter may vote "yes," abstain, or vote "no," the outcome is "yes" or "no," and all voters play interchangeable roles. The extension to symmetric (j,2) simple games, in which each voter chooses from among $j$ ordered levels of approval, also models some natural decision rules, such as pass-fail grading systems. Each such game is determined by the set of (anonymous) minimal winning profiles. This makes it possible to count the possible systems, and the counts suggest some interesting patterns. In the $(3,2)$ case, the approach yields a version of May's Theorem, classifying all possible anonymous voting rules with abstention in terms of quota functions. In contrast to the situation for ordinary simple games these results reveal that the class of simple games with 3 or more levels of approval remains large and varied, even after the imposition of symmetry.
\end{abstract}

Keywords: Anonymity, Abstention, Games with several levels of approval $(j, k)$ games Absolute and simple strict majority rule, Grading systems

\section{Introduction}

Our initial concern is with voting systems in which each voter casts a "yes" or "no" vote, or abstains, and the outcome is a collective "yes" or "no." If we treat abstention as a level of approval intermediate to "yes" and "no," and further assume that all voters play interchangeable roles (the voting system is anonymous), what voting systems are possible? Does the broad range of theoretical possibilities for such systems suggest any alternatives to the more limited collection that are typically used in real voting? What happens to these questions if we further generalize by allowing each voter to express any one of $j$ ordered levels of approval? Which real-world decision-making systems are modeled by this generalization?

To accommodate voting systems with these features, we must make two changes to the mathematical models known as simple games; we need to generalize, by allowing multiple levels of approval by voters, and also need to specialize, by considering only models that are symmetric, reflecting the anonymity of the voting system being modeled. The goal of the paper is to analyze the joint effect of these two changes on the resulting class of models. In this respect, Côrte-Real and Pereira's (2004) paper is most closely related to this one. Nonetheless, the overlap in content is small.3 Considerably more work has been done on the separate effects of these two changes. The generalization to multiple levels arose out of the observation that, while many real voting systems allow voters to abstain (or be absent), simple games, by their nature, cannot take this possibility into account; those who do not vote "yes" are presumed to vote "no." See, for example Bolger (1986), Fishburn (1973), Rubenstein (1980). More recently, there has been considerable attention (in, for example, Amer et al., 1998; Bilbao, 2000, Bilbao et al., 2008a, 2008b; Bolger, 1993, 2000; Felsenthal and Machover, 1997, 1998; Freixas, 2005a, 2005b; Lindner, 2005; Tchantcho et al., 2008) paid to the question of how to modify various power indices so as to take account of abstention. In Freixas and Zwicker (2003), we introduce a class of structures - the (j,k) simple games, or ( $j, k)$ games, for short that generalize simple games by allowing $\mathrm{j}$ ordered levels of approval in the input and $\mathrm{k}$ ordered levels in the output. In that paper, a definition of weighted voting for ( $j, k)$ games is proposed, based on a natural generalization, grade trade robustness, of the combinatorial characterization of weighted voting for simple games in terms of trade robustness (see Taylor and Zwicker, 1992, 1999). 
Somewhat surprising is that the resulting definition of weighted voting with abstention is strictly more general than what one might first surmise. As we see in the current paper, this added generality persists even for anonymous voting rules, in which all votes count the same.

The anonymous voting rules we study here are precisely the same as what one obtains by imposing symmetry on (j,k) games in general. We take the simpler approach here, however, of defining and analyzing anonymous $(j, k)$ voting rules without drawing any formal connection to $(j, k)$ games.4

Even without added levels of approval, standard simple games can be very complicated. Their specialization to symmetric simple games, however, are simple indeed; each such game corresponds to qualified majority rule, in which an issue is passed if and only if the number voting in favor meets or exceeds some threshold or quota, q. We refer to this result as May's Theorem for Simple Games, though the better term might be May's Theorem "with bias." May's original result (see May, 1952 or Taylor and Pacelli, 2008) considers only anonymous voting systems (for two alternatives) that are neutral: there is no built-in bias towards "yes" or "no" as outcomes, so that they are treated symmetrically. Neutrality is a natural assumption when the alternatives are two candidates for an elected position, and the voting system is intended to present a level playing field. However, when the alternatives are yes (yes, change the status quo by passing a certain new law or proposal) and no (no, reject the proposal and leave the status quo unchanged) then a built in bias is often seen as appropriate. For example, a strong bias towards "no" is common in voting systems for amending constitutions. Our interest here is more in this area of legislative voting systems. Neutral voting systems for two alternatives correspond to constant-sum simple games, and the original version of May's Theorem may be stated as follows: for each odd value of $n$, there is, up to isomorphism, a unique simple game that is both constant-sum (also called decisive) and anonymous - it corresponds to standard majority rule (in which $q$ is exactly half of $n+1$ ).

There seem to be several natural directions ways to extend May's Theorem to the context of abstention and, more broadly, to the context of multiple levels of approval. This paper is concerned primarily with one of these, in the form of a classification of all anonymous $(3,2)$ voting rules via quota functions and a different classification of all anonymous $(j, 2)$ voting rules via the set of minimal anonymous profiles. Absolute majority rule, simple strict majority rule, as well as several variations that employ supermajorities, quorums, or what we term soft quorums are all examples that fit into the

$(3,2)$ context. It turns out that grading systems provide natural examples of voting systems with additional levels of input approval. Each of several tests can be thought of as a voter, with the score achieved (by some individual student enrolled in a course) being the vote. The election outcome is that the student either passes the course, or fails. We provide examples of such systems using the mean and others using the median - in fact, one part of the procedure to get the driving license in Spain can be seen as an example of the latter. Although it is not our main focus here, it would be quite natural as well to consider anonymous $(j, k)$ voting rules for which the number $k$ of levels of output approval exceeds 2; natural examples include grading systems that provide more than 2 possible final course grades.

An alternate extension of May's Theorem for Simple Games is suggested by the following reformulation of the original version: every anonymous hypergraph that is monotonic is weighted. A hypergraph is a simple game with the monotonicity requirement suspended; precise definitions appear in Freixas and Zwicker (2003). This precise reformulation fails to extend; nonweighted anonymous $(3,2)$ games exist. However, there is a strong form of monotonicity that does imply weightedness for anonymous $(3,2)$ games, and this statement may also lay claim to being the extension of May's Theorem to three levels of approval. In Zwicker (2009), we explore this alternate extension, providing several equivalent characterizations of weightedness and several parameterizations of the class of weighted $(3,2)$ games, and we discuss some of the implications for real voting systems. In fact, one cannot completely avoid the topic of weightedness in the current paper, as so many of the natural examples prove to be weighted.

The rest of this paper is organized as follows. We begin, in Section 2, with a brief presentation of several examples of real and fictive anonymous voting systems, described in nontechnical language. In Section 3 we give precise definitions for anonymous $(j, k)$ voting rules, point out that any such rule $G$ is determined by a set $M(G)$ of minimal anonymous profiles, and revisit the examples in 
light of these developments. Section 4 takes up our first extension of May's Theorem to the context of anonymous voting with abstention.

It turns out that in May's Theorem with Abstentions I the role of a quota is replaced by that of a quota function, $q(a)$, where a stands for the number of abstaining voters. Monotonicity places some conditions on the allowable quota functions, and this version of May's Theorem then states that anonymous $(3,2)$ voting rules correspond exactly to quota functions satisfying these conditions. The $M(G)$ method allows us to construct a table (see Section 5) that enumerates the number of anonymous $(j, 2)$ voting rules with $n$ voters, for selected values of $j$ and $n$. The table suggests two patterns, which we have been able to confirm. First, we show that the number of anonymous $(3,2)$ voting rules is $2^{n+1}$. Second, we prove a form of duality, asserting that every anonymous ( $j$, 2) voting rule with $n$ voters corresponds to an anonymous $(n+1,2)$ voting rule with $j-1$ voters.

In Section 6 we briefly sketch an extension of our methods that handles the case of $(j, k)$ voting rules with more than 2 levels of output approval. The concluding Section 7 returns to the matter of describing real voting practice adequately via simple mathematical models, sounding a cautionary note.

\section{Examples}

The following examples, described here informally and later revisited, inform much of what we do throughout the paper. Note that all the voting systems we model have an assembly with some fixed number $n$ of voters5; when $n$ is unspecified we are discussing a class of related voting systems, one for each value of $n \geq 1$.

In the first group, an alternative (such as a motion, a bill, or an amendment) is pitted against the status quo and players or voters are allowed to vote in favor or against, with abstention or absence allowed as a middle option.

\subsection{Absolute majority rule}

An alternative to the status quo is passed according to absolute majority rule if more than half of the assembly votes in favor. Note that in this system absence, abstention, and voting "no" are all equivalent. Absolute majority rule is thus an example of a rule that can be modeled by a (classical) $(2,2)$ simple game. In the spirit of the current paper, however, we will count both absence and abstention in the "middle level" of approval.

In qualified absolute majority rule, some fixed absolute number of "yes" votes are needed for passage, and this threshold may be expressed as some fraction of the voting body.

\subsection{Simple (or "relative") majority rule}

The alternative defeats the status quo by simple (strict) majority rule if the number of players voting in favor of it is greater than the number voting against. Abstention and absence are equivalent, and fall in the middle approval level.

This rule may be the most natural extension possible of majority rule to a context allowing abstention but forbidding tied election outcomes. While this context is incompatible with the full neutrality axiom, we can come close.

May's Theorem with Abstention (third version) considers anonymous and monotonic voting rules in which voters may vote yes (favoring some proposed alternative to the status quo) or no, or may abstain, and the outcome is either collective approval of the alternative, or its rejection. Simple strict majority rule is the unique such rule that is "almost neutral."

Here, a rule is almost neutral if the election outcome reverses whenever each voter reverses her vote (with abstain taken to be its own reverse), except when such a reversal yields the identical profile. For this exceptional profile, the status quo is retained. Note that if we assume the number of voters is odd and no one abstains, this version yields May's original result as a special case. 


\subsection{Voting using ratio and difference quotas}

In a ratio quota system, the alternative defeats the status quo if the number voting in favor is at least equal to some specified multiple quota $m$ of the number voting against; equivalently, the fraction of "active" voters (that is, voters who are present and vote "yes" or "no") who vote in favor must be at least equal to the corresponding ratio quota $r$, where $r=m^{6} /(1+m)$. For example, passage might require that at least twice as many vote in favor as vote against (so that $m=2$ ); equivalently, the fraction of active voters who vote in favor must be at least $2 / 3$. The term qualified simple majority might also be applied to this type of system.

In a difference quota system, the alternative defeats the status quo if the number voting "yes" exceeds the number voting "no" by at least some specified absolute amount, or difference quota, d.

If all voters are active, there is no distinction, of course, between ratio quota systems and difference quota systems, while absolute majority rule coincides with simple majority rule. When abstentions or absences are possible, however, ratio quotas are quite different from difference quotas. Simple majority rule is, to all intents and purposes, both a ratio quota system and a difference quota system, and absolute majority rule is neither a ratio quota system nor a difference quota system.

\subsection{Treaty ratification in the U.S. Senate}

Ratification of a treaty by the Senate of the United States requires that at least two-thirds of those present approve of the treaty, subject to the requirement that more than half of all Senators be present at the vote. Notice that this example actually differs from the previous one in two ways. First, there is a quorum in the form of a requirement that at least 51 (of the total of 100) Senators be present for the vote. This requirement forces us to treat absence differently from abstention. Second, the wording implies that abstention is equivalent to a "no" vote, which was not the case in the previous example. Thus, we will record an absence as a middle level of approval, with abstention and "no" votes both counting in the lowest of the three approval levels.

As we would expect, a "no" vote in this system is often more negative than an absence. For example, with 60 Senators voting "yes," 30 voting "no" and 9 absent, a "no" by the 100th Senator (whom we assume to be in opposition) defeats a proposed treaty, while her absence would result in approval. As a consequence of the quorum, however, the effects can be reversed; with 40 Senators voting "yes," 10 voting "no," and 49 absent, the 100th Senator would be advised to stay away, rather than appear and vote "no." This perverse consequence of a quorum has had real political consequences, in addition to the theoretical ones discussed briefly in Section 4.2 (and also in Freixas and Zwicker, 2003).

\subsection{Majority threshold}

As discussed in Côrte-Real and Pereira (2004), for certain types of referenda in Germany, Hungary, and Denmark the alternative defeats the status quo provided that the number voting in favor be greater than the number voting against, subject to the additional requirement that more than a certain fixed fraction, called the majority threshold (and equal to 1/4 in Hungary, for example), of the registered voters vote in favor. Interestingly, a majority threshold requirement has a qualitatively different effect from that of a quorum. In particular, it avoids the problem of nonmonotonicity, and so does not open the door to manipulation.

The majority threshold systems that we know of are used in national referenda, rather than in legislatures. There are additional ways in which studies of referendum voting seem to lead to somewhat different conclusions from those of legislative voting. For instance, the distinction we have drawn between abstention and absence becomes irrelevant in referendum voting.

Yet another difference is that for countries whose referendum voting rules employ a true quorum rather than a majority threshold, the resulting problem of manipulation seems to be more serious, in 
practice, than it is with legislative systems.9 For example, article 75 of the Italian constitution allows for abrogative referenda, in which a majority of the participating voters can overturn all, or part, of a law by voting "yes," provided that a quorum of $50 \%$ or more of the registered voters participates by casting a vote. Uleri (2002) argues that in the 1990s the "strategic option" of "campaigns of effective demobilization" evolved to become the dominant tool used by Italian political parties to defeat article 75 referenda. In some cases, as with Berlusconi in 2000, the prime minister "openly invited electors to stay away from the polling stations." The author concludes that in such referenda the "nonvoters are more equal than the voters," and that "the existence of a voter quorum distorts free and democratic competition." In this connection, see the comments in Côrte-Real and Pereira (2004) on the recommendations of the European Commission for Democracy through Law. As we see in the next example, however, majority thresholds do not represent the only possible solution to the problem of imposing voter participation floors while avoiding the perverse effects of a quorum. Variations on the above five examples can be found in national legislative bodies, town councils, etc. used throughout the world.10 The next example, however, is a bit different.

\subsection{Soft quorum}

The alternative defeats the status quo, with a Soft quorum, providing that the number voting in favor be at least equal to the sum of one-third of the total assembly plus an additional amount equal to the number of those present who do not vote in favor - equivalently, that the number be at least equal to the sum of one-third of the total assembly plus one-half of any of those who are present in excess of the one-third.

To our knowledge, no voting system similar to this has ever been used. What recommends its consideration? At one extreme, notice that if all members are present and two-thirds vote in favor of an alternative to the status quo (with the other one-third voting "no," or abstaining), then the sum of one-third plus half of two-thirds is exactly two-thirds, and the alternative is (barely) approved, according to the rules stated above. At the other extreme, the absolute minimum number of "yes" votes that can ever succeed in approving the alternative is one-third of the assembly.

In particular, suppose we consider a 100 member Senate, and compare our Soft quorum system with the (actual) treaty ratification system of Example 2.4. In either system, with all members present, a minimum of 67 votes is required for ratification, while with members absent the absolute fewest number of "yes" votes that can ever succeed in ratifying a treaty is 34. Thus, while the Soft Quorum system differs from Example 2.4 in some important respects, it achieves at least some of the same effects, and it does so without any of the perverse consequences of the more standard, "hard" quorum.

The remaining examples are devoted to games with more than three levels of approval allowed to voters.

\subsection{Voting on a promotion}

This example is adapted from Hsiao and Raghavan (1993, p. 241), who describe a committee that must decide on the promotion of a certain junior colleague in a mathematics department. The original example was not anonymous: it assumed two types of voters, with different levels of influence. In our variation, a committee has 4 members, each of whom may express strong support, marginal support, weak opposition, or strong opposition. The candidate will be approved exactly when all three of the following conditions are met:

(1) Nobody shows strong opposition.

(2) One or more members shows strong support.

(3) Two or more members show at least marginal support. 


\subsection{A mean grading system}

At some colleges and universities, each student enrolled in a course is typically evaluated several times. For the sake of simplicity, we assume that each student takes several tests, and that each test is assigned an integer score between 0 and 10, inclusive. Some rule is used to amalgamate these individual scores, and the result is a final course mark (also called a course score or course grade).

Perhaps the most commonly used amalgamation method for course grades is a weighted mean (average). A student's course mark may then be termed her or his course average. Often, this mark is compared to some threshold, which determines whether the student passes or fails the course. In some cases, only this ultimate pass-fail determination is entered in the student's record.

Any such grading scheme corresponds to an $(11,2)$ simple game; we may think of each test as a "voter," voting on the issue, "should this particular student pass the course?" To achieve anonymity, we will assume that all evaluations count equally (for example, all weights are equal in the weighted mean), which is not always true in practice. We will somewhat arbitrarily set a threshold of 5 for passing the course.

Suppose that in a certain Statistics class there are 7 tests. Josephine's seven grades are 10, 10, 10, 4 , 4,4 , and 4, while Eric's grades are 5, 5, 5, 5, 0, 0, and 0. Then Josephine's course average is $6.714 \ldots$ while Eric's is $2.857 \ldots$. . so that Josephine passes the course, while Eric fails.

\subsection{A median grading system}

Assume that a certain Engineering class has a set-up exactly like that of the previous example, with only one change: the amalgamation method used to determine a student's final mark is the median, rather than the mean, applied to a student's seven test scores. If Josephine and Eric have exactly the same individual test scores in Engineering as in Statistics, then Josephine's Engineering course mark is 4, so she fails Engineering, while Eric's course mark is 5, so he passes Engineering.

Of course, these distributions of marks are extreme and unlikely in practice. But the systems will be useful in illustrating the classification approach we provide in the next section.

\subsection{Getting a driving license in Spain}

One of the requirements for obtaining a license to drive cars or motorbikes in Spain is to pass a theoretical examination. This exam is divided into three parts: assessing knowledge of traffic signals, marks, etc.; of car mechanics; and of civil behavior while driving, respectively. Each part has 10 questions. Passing the theoretical exam requires a minimum of 8 correctly answered questions on each part.

As in the previous two examples, the three parts of the test may be considered to be the three "voters." We will see later that this example represents, in one sense, a type of median.

\section{Anonymous voting rules with multiple levels of approval}

\section{1. Anonymous profiles and voting rules}

Let $n, j$, and $k$ be positive integers. Consider a voting rule $G$ for $n$ voters, in which each voter records her or his preference in the form of a choice of input approval level, chosen from the $j$ ordered levels available, and in which the result of the election is a (collective) choice of output approval level, chosen from the $k$ ordered levels available. If we further assume that $G$ is anonymous, so that election results depend solely on the number of voters who select each possible approval level, rather than on their individual identities, then the information that must be made available to $G$ may be recorded in the form of a vector, as follows. 
Definition 3.1. An anonymous profile for an $n$-voter anonymous $(j, k)$ voting rule consists of a vector $p$ $=(1 p, 2 p, \ldots, j p)$ of $j$ nonnegative integers that sum to $n$. We will use $P(n, j)$ to denote the set of all such vectors. In the special case $j=3$ we will typically write $p=(y, a, h)$.

Here, $y=1 p$ denotes the number of "yes" voters, $a=2 p$ denotes the number who abstain (or are absent), and $h=3 p$ denotes the number who vote "no." More generally we intend that level 1 represents the strongest level of approval, and level $j$ the weakest, with $i p$ denoting the number of voters who vote for the ith level of approval for $i=1, \ldots, j$. As all of our profiles will be anonymous, we will typically refer to any such vector as a "profile."

Notice that if some single voter were to change her vote by strengthening her approval level from $i$ to $i-1$, the effect would be a change from profile $p$ to profile $p$ ', in which "a 1 shifts left"; we will say that $p^{\prime}>1 p$ when:

$$
{ }_{h} p^{\prime}= \begin{cases}i-1 p+1, & \text { if } h=i-1 \\ i p-1, & \text { if } h=i \\ h p, & \text { otherwise. }\end{cases}
$$

Definition 3.2. We will use $\delta$ to denote the partial order on $P(n, j)$ obtained as the transitive closure of the $>_{1}$ relation.

The $\delta$ order is commonly known as the "left shift order." In our intended application, p' $\delta p$ holds if and only if the profile $p^{\prime}$ may be obtained from $p$ by having one or more voters from $p$ change their votes by approving at a (strictly) stronger level. Thus $\delta$ may be thought of as a form of dominance, and when $p^{\prime}$ dominates $p$ we expect the resulting level of output approval for $p^{\prime}$ to be at least as strong as that for $p$.

Definition 3.3. Let $K=\{0,1, \ldots, k-1\}^{11}$. A function

$$
V: P(n, j) \rightarrow K
$$

is monotonic if for every $p, p^{\prime} \in P(n, j)$, if $p^{\prime} \delta p$ then $V\left(p^{\prime}\right) \geq V(p)$. Given positive integers $n, j$, and $k$, an anonymous $(j, K)$ voting rule for $n$ voters is a pair $G=(n, V)$ in which $V: P(n, j) \rightarrow K$ is monotonic

\section{Table 1}

\begin{tabular}{|c|c|c|c|c|c|}
\hline $\bar{b}$ & $\left(\begin{array}{lll}3 & 0 & 0\end{array}\right)$ & $\left(\begin{array}{lll}2 & 1 & 0\end{array}\right)$ & $\left.\begin{array}{llll}2 & 0 & 1\end{array}\right)$ & $\left(\begin{array}{lll}1 & 2 & 0\end{array}\right)$ & $\left(\begin{array}{lll}1 & 1 & 1\end{array}\right)$ \\
\hline$\left(\begin{array}{lll}1 & 0 & 2\end{array}\right)$ & $\left(\begin{array}{lll}0 & 3 & 0\end{array}\right)$ & $\left(\begin{array}{lll}0 & 2 & 1\end{array}\right)$ & $\left(\begin{array}{lll}0 & 1 & 2\end{array}\right)$ & $\left(\begin{array}{lll}2 & 0 & 1 \\
1 & 2 & 0\end{array}\right)$ & $\left(\begin{array}{lll}2 & 0 & 1 \\
0 & 3 & 0\end{array}\right)$ \\
\hline$\left(\begin{array}{lll}1 & 1 & 1 \\
0 & 3 & 0\end{array}\right)$ & $\left(\begin{array}{lll}1 & 0 & 2 \\
0 & 3 & 0\end{array}\right)$ & $\left(\begin{array}{lll}1 & 0 & 2 \\
0 & 2 & 1\end{array}\right)$ & $\left(\begin{array}{lll}0 & 0 & 3\end{array}\right)$ & & \\
\hline
\end{tabular}

Matrices for all anonymous $(3,2)$ voting rules with 3 players.

\subsection{Classifying $(j, 2)$ rules via minimal winning profiles}

Our principal concern here is with the case $k=2$, for which some specialized terminology is appropriate.

Definition 3.4. Let $G=(n, V)$ be an anonymous $(j, 2)$ voting rule for $n$ voters, and $p \in P(n, j)$. If $\vee(p)=$ 1 , we say that $p$ is a winning profile, and if $V(p)=0$, then $p$ is a losing profile. If $p$ is a winning profile and if for every profile $p^{\prime} \in P(n, j)$ with $p \delta p^{\prime}, p^{\prime}$ is a losing profile, then $p$ is a minimal winning profile. We will use $\wedge p$ to denote $\{q \in P(n, j) \mid q \delta p$ or $q=p\}$, the cone over $p$, and $M(G)$ will denote $\{p \in P(n$, j) $\mid p$ is a minimal winning profile of $G\}$.

The only demand we have placed on an anonymous $(j, k)$ voting rule is that the function $V$ be monotonic. When we restrict ourselves to the case $k=2$ one effect is to admit two harmless (if not 
particularly useful) voting rules: the rule for which every profile is winning, and the rule for which no profile is winning. Winning profiles and minimal winning profiles play roles similar to those of winning coalitions and minimal winning coalitions in the theory of simple games. It is easy to see that for anonymous $(j, 2)$ voting rules, monotonicity is equivalent to the assertion that whenever $p$ is a winning profile, every $p^{\prime} \epsilon \wedge p$ is also winning. Moreover, given the set $M(G)$ of minimal winning profiles for $a(j, 2)$ game, we can easily recover the game $G M$, because the value function satisfies $\vee(q)=1$ if and only if $q \in U_{p \in M(G) \wedge p}$.

In practice, it is useful to present $M(G)$ in the form of a matrix, $M(G)$, in which each row enumerates a different profile $p=(1 p, 2 p, \ldots, p)$ in $M(G)$ and the rows are listed in descending lexicographic order. Thus each anonymous ( $j, 2)$ voting rule for $n$ players yields either an empty matrix, for which all the profiles lose, or a unique matrix $M(G)$ having the following four properties:

(i) each row has length $j$,

(ii) each row has sum $n$,

(iii) the rows are distinct and incomparable in the $\delta$ order, and

(iv) the rows are listed in descending lexicographic order.

Conversely, it is easy to see that each matrix $M$ with these four properties is equal to $M(G)$ for a unique anonymous ( $j, 2$ ) voting rule $G$ for $n$ players.

Consequently, given any $j$ and $n$ we can generate and count all anonymous $(j, 2)$ voting rules for $n$ players. As an example we provide in Table 1 the complete list of matrices corresponding to anonymous $(3,2)$ voting rules for $n=3$.

\subsection{Examples, revisited}

Notice for example that matrices

$$
\left(\begin{array}{lll}
2 & 0 & 1
\end{array}\right) \quad\left(\begin{array}{lll}
2 & 0 & 1 \\
1 & 2 & 0
\end{array}\right)
$$

correspond to absolute majority rule and to simple strict majority rule respectively (Examples 2.1 and 2.2). The matrix for the particular soft quorum Example 2.6, with a voting body of size $n=10$, is

$$
\left(\begin{array}{lll}
7 & 0 & 3 \\
6 & 2 & 2 \\
5 & 5 & 0
\end{array}\right)
$$

The matrix associated to the rule from Example 2.7 is

$$
\text { (1 } 1220)
$$

For the "median" examples described in 2.9 and 2.10 the matrices are

$$
\text { (00000400003) }
$$

and

$$
\text { (00300000000) }
$$

respectively.

The above examples suggest that the matrix associated to a simple voting system is a simple matrix, but this is not always the case. To illustrate this, let us consider the exercise of determining the matrix associated to the mean grading system in Example 2.8. It is straightforward to see that this problem reduces to obtaining all the integer solutions for the equation 


$$
\sum_{i=1}^{7} x_{i}=35, \text { where } 10 \geqslant x_{1} \geqslant x_{2} \geqslant \cdots \geqslant x_{7} \geqslant 0 .
$$

Then, each solution $(x 1, \ldots, x 7)$ provides a row $p$ of $M(G)$ and vice versa, as follows. We initially think of $p$ as having all components null. Then if $x_{i}=b$ where $0 \leq b \leq 10$, we add one unit to the $(11-b)$ th component of $p$, and do so for each component $i$ to get $p$. For instance, if $x=(10,8,7,3,3,2,2)$ then $p=(1,0,1,1,0,0,0,2,2,0,0)$. Finally, consider all the transformed solutions in lexicographic order to get $M(G)$.

Unfortunately, there are 734 solutions (obtained with the help of a computer program), which might seem to suggest that the matrix is not very useful. However, even in this case, these 734 minimal winning profiles correspond to 908,775 minimal nonanonymous profiles (wherein two profiles are considered to be the same only when each individual voter votes the same way). The latter number is obtained by considering the number of integer solutions of the equation

$$
\sum_{i=1}^{7} x_{i}=35 \text {, where } 0 \leqslant x_{i} \leqslant 10 \text {, for each } i \text {. }
$$

In fact, it is easy to show that this matrix, as well as any matrix $M$ similarly arising from a game in which a mean level of approval is measured against a fixed threshold, has as many rows as it possibly can - the rows form a maximal antichain in the $\delta$ order. Thus, it is impossible to add any additional row to $M$ without violating the requirement that the rows be incomparable in the $\delta$ order. In one sense, then, the mean grading systems are as far as possible from the median systems, which have one-row matrices.

\subsection{Weighted ( $j, k)$ voting rules}

How should "weighted voting" be defined, in the context of voting with abstention or, more broadly, when there are multiple levels of input and output approval? The answer we proposed for the nonanonymous case in Definition 2.5 of Freixas and Zwicker (2003) went roughly as follows: each individual player $p$ is endowed with a vector $w(p)=\left(w_{1}(p), \ldots, w_{j}(p)\right)$ of $j$ different weights. If that player's chooses to approve at level $i$, she contributes the amount $w_{i}(p)$ to the total weight $T$ cast. A vector of $k-1$ real number quotas $q_{1} \leq q_{2} \leq \cdots \leq q_{k}-1$ then determines the collective outcome approval level to

be the greatest "i" for which $T \geq q_{i}$. In that paper, we justified our definition by showing (Theorem 4.4) that the standard trade robustness characterization of weighted simple games (Taylor and Pacelli, 2008; Taylor and Zwicker, 1992, 1999) has a natural generalization, called grade trade robustness, to the context of many levels of approval, and that a $(j, k)$ simple game or hypergraph is grade trade robust if and only if it satisfies Definition 2.5. Here is the version for the anonymous case:

Definition 3.5. Let $G=(n, \vee)$ be an anonymous $(j, k)$ voting rule for $n$ voters. A representation of $G$ as a weighted anonymous $(j, k)$ voting rule consists of a vector $w=\left(w_{1}, \ldots, w j\right)$ of $j$ real number weights, together with a vector $q=\left(q_{1}, \ldots, q_{k-1}\right)$ of $k-1$ real number quotas satisfying $q_{1} \leq q_{2} \leq \cdots \leq$ $q k-1$ such that for every profile $p$ and every $r$ with $0 \leq r \leq k-1, V(p)=r$ if and only if $q_{r} \leq w(p)<q_{r+1}$, where $w(p)$ denotes the inner product $w \cdot p$ and we think of $q_{0}$ and $q_{k}$ as having nominal values of $-\infty$ and $+\infty$ respectively.

We say that $G=(n, V)$ is a weighted anonymous $(j, k)$ voting rule if it has such a representation.

This paper is limited almost exclusively to the case $k=2$, so we need only a single quota. Notice that our definition here is essentially the same as the version in Freixas and Zwicker (2003), modified only by the requirement that all players be endowed with identical weight vectors. While this appears to be the obvious thing to do, two points are worth emphasizing. First, the resulting notion of weighted voting is strictly more general than the naive version that might first come to mind. This is so even for the simplest case we consider - that of anonymous (3,2) voting rules in which voters may vote "yes," 
"no," or abstain, and the outcome is collective approval or disapproval of a motion - where the naive notion corresponds to the ratio quota systems of Example 2.3 (see further discussion in Section 3.5).

Second, it is straightforward to show that the definition above inherits the combinatorial justification provided in Freixas and Zwicker (2003) for the nonanonymous case; an anonymous ( $j, k$ ) voting rule is weighted according to Definition 3.5 if and only if it is grade trade robust.One might hope, however, that in the anonymous case the full strength of grade trade robustness would not be required for weightedness. Quite recently, Zwicker (2009) shows that for the $(3,2)$ anonymous case, weightedness (or grade trade robustness) is equivalent to a strong form of monotonicity called bimonotonicity.

If an anonymous $(j, k)$ voting rule for $n$ voters is weighted then it admits an infinite number of weighted representations as a weighted anonymous $(j, k)$ voting rule. This allows us to adjust a given representation in a variety of ways, so that it achieves some desired form or so that the weights reflect more directly certain properties of the rule. In particular, any anonymous $j, k$ ) voting rule can be given a weighted representation in which

(1) The common weight vector $w$ satisfies $w 1 \geq w 2 \geq \cdots \geq w j$.

(2) All individual weights and quotas are integers.

(3) Any desired weight $w_{i}$ is zero, or all weights are positive.

For example, any anonymous $(3,2)$ rule that is weighted has a weighted representation in which the weight vector $w=$ (Wyes, Wabstain, $\left.W_{n o}\right)$ consists of integers satisfying $w_{y e s} \geq 0, w_{a b s t a i n}=0$, and $W_{n o} \leq 0$, and the quota is an integer.

\subsection{Examples, revisited again}

What can we now say about our examples, in light of the notion of weighted voting just introduced? The perverse effect of the quorum violates monotonicity in Example 2.4 (as we show later in Section 4.2), the rule for ratifying treaties in the U.S. Senate, and thus guarantees that this system cannot be modeled by an anonymous $(3,2)$ voting rule at all, let alone by a weighted rule. Example 2.5 , the referendum with a majority threshold, is monotonic, but it turns out not to be weighted, as we point out in Section 4.2; in fact, as far as we know such referenda provide the only naturally occurring examples of nonweighted anonymous $(3,2)$ voting rules. Each of the remaining examples from section 3.5 is modeled by an anonymous weighted $(j, 2)$ voting rule, as we now show.

(i) Absolute majority rule, Example 2.1, is modeled by an anonymous (3,2) voting rule, in which

$$
V(A)=V(y, a, h)= \begin{cases}\text { win } & \text { if } y>a+h, \\ \text { lose } & \text { otherwise. }\end{cases}
$$

A weighted representation for it is

$$
q=\left[\frac{n}{2}\right]+1, \quad w=(1,0,0) .
$$

An equivalent representation is

$$
q=1, w=(1,-1,-1) \text {. }
$$

(ii) Example 2.2, simple majority rule, corresponds to a different anonymous (3, 2) voting rule:

$$
V(p)=V(y, a, h)= \begin{cases}\text { win } & \text { if } y>h, \\ \text { lose otherwise. }\end{cases}
$$

It is straightforward to obtain

$$
q=1, w=(1,0,-1) \text {, }
$$


as a weighted representation.

(iii) For Example 2.3, with a multiple quota of $m=2$ and a ratio quota of $r=2 / 3$, we have:

$$
V(p)=V(y, a, h)= \begin{cases}\text { win } & \text { if } y \geqslant \frac{2}{3}(y+h), \\ \text { lose } & \text { otherwise. }\end{cases}
$$

A weighted representation is

$$
q=0, w=(1,0,-2) \text {. }
$$

(iv) A weighted representation for the soft quorum of Example 2.6, with a voting body of size $n$, is

$$
q=n, w=(3,0,-3)
$$

(v) A weighted representation of Example 2.7 (the modified version of Hsiao and Raghavan's system for voting on promotions), modeled as an anonymous $(4,2)$ voting rule, is

$$
q=37, w=(11,8,7,0) \text {. }
$$

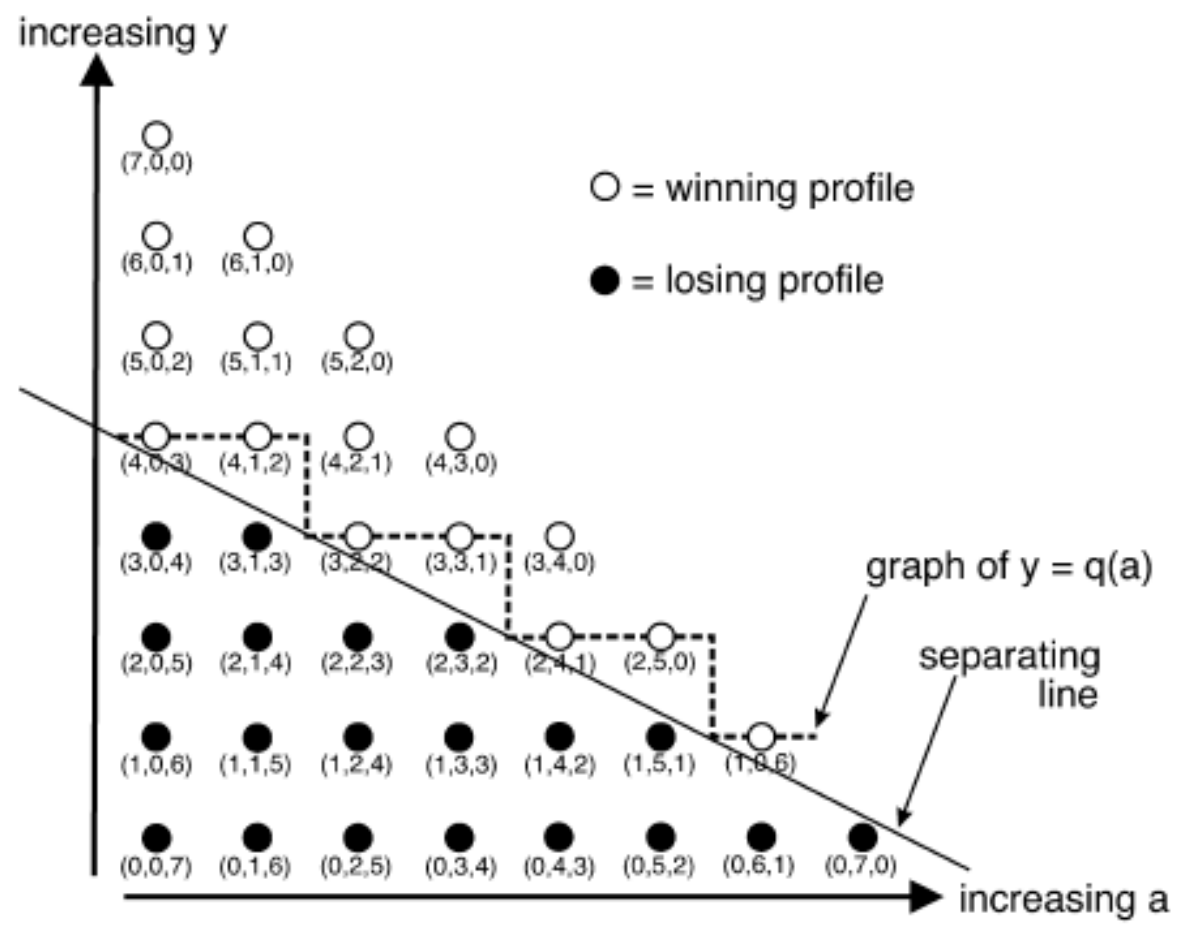

Fig. 1. Simple majority rule, with abstention, for 7 voters,

(vi) Our two examples of grading systems, Examples 2.8 and 2.9, are nicely modeled by weighted anonymous $(11,2)$ voting rules. It is not difficult to see that the following is a representation:

$$
\begin{array}{ll}
q_{\text {median }}=4, & W_{\text {median }}=(1,1,1,1,1,1,0,0,0,0,0), \\
q_{\text {mean }}=35, & W_{\text {mean }}=(10,9,8,7,6,5,4,3,2,1,0) .
\end{array}
$$

(vii) Example 2.10, the procedure for getting the driving license in Spain, is also modeled as an (11, 2) voting rule, with a weighted representation similar to that just described for the median: 


$$
q=3, \quad w(p)=(1,1,1,0,0,0,0,0,0,0,0) .
$$

Because of the similarity to the weighted representation for the median grading system just given, our inclination is to think of 2.10, as well, as a type of median. In fact, each may be described by choosing an integer $r$ with $1 \leq r \leq n$ together with a level $s$ of approval and requiring that the approval level of the $r$ th voter (when voters are listed in any order with nonascending strength of approval) approve at a level of $s$ or stronger.

\section{Quota functions and anonymous $(3,2)$ rules}

Recall that in the special case $j=3, k=2$, an anonymous voting rule $G$ can be thought of as one in which each voter casts a vote of "yes," or of "no," and also has the option of abstaining; we represent a profile as an ordered triple $(y, a, h)$ of nonnegative integers, and the result of applying the rule $G$ to such a profile is that the proposal before the assembly is either approved or turned down. In this special case, a geometric depiction of the set $P(n, 3)$, and of a partition of this set into winning and losing profiles, sheds some additional light. The elements of $P(n, 3)$ correspond to the integer points $(y, a, h)$ of $R^{3}$ on the section of the plane $y+a+h=n$ contained in the first octant $(y \geq 0, a \geq 0$, $h \geq 0$ ). This section forms an equilateral triangle.

For our purposes, it is helpful to project the integer points of this triangle onto the $x y$ plane in a way that slightly distorts this triangle; the xy coordinates of $(y, a, h)$ will be $(a, y)$ - that is, the $x$-coordinate represents the number of abstentions, and the $y$-coordinate represents the number of "yes" votes. After plotting the representations of all points of $P(7,3)$ in this way, we obtain a selection of points from a rectangular grid, which occupy a region in the form of a right triangle. Each node $(a, y)$, in Fig. 1, is labeled with its original coordinates $(y, a, h)$. The figure also illustrates Example 2.2 (simple strict majority rule) for 7 voters; winning profiles are plotted as empty circles while losing ones show as filled, black circles. In simple strict majority rule, $(y, a, h)$ is winning when $y>h$ and losing when $y \leq h$. Notice that the two regions are separated by the graph of a certain step function, $y=q \operatorname{ssm}(a)$. In the case of Fig. 1, the graph is drawn as a dashed line; qssm has domain $\{0,1,2,3,4,5,6\}$ and satisfies

$$
\begin{aligned}
& q_{S S M}(0)=4=q_{S S M}(1), \quad \quad q_{S S M}(2)=3=q_{S S M}(3) \text {, } \\
& q_{S S M}(4)=2=q_{S S M}(5), \quad \text { and } q_{S S M}(6)=1 \text {. }
\end{aligned}
$$

We will refer to any figure showing this grid and separating step function as a quota function diagram. The value $q(a)$ is the minimum number of "yes" votes needed for collective approval, expressed as a function of the number a of abstentions. If there is some number a of abstentions for which collective approval is unobtainable even when all the nonabstainers vote "yes," then this value a fails to be in the domain of $q$.

An alternative to simple strict majority rule would be absolute majority rule, Example 2.1. With 7 voters, $(y, a, h)$ would be winning exactly when $y \geq 4$. The quota function would then have domain $\{0$, $1,2,3\}$ and be given by

$$
q_{S S M}(0)=q_{S S M}(1)=q_{S S M}(2)=q_{S S M}(3)=4 \text {. }
$$

A quota function diagram (omitted) would show the graph as a horizontal line segment.

\subsection{May's Theorem with abstention}

Definition 4.1. For any anonymous $(3,2)$ voting rule for $n$ voters $G=(n, V)$, the induced quota function $q_{G}$ is given by $q_{G}(a)=$ the least value of $y$ such that $(y, a, n-a-y)$ is a winning profile, if such a $y$ exists, with $q_{G}(a)$ undefined otherwise.

Clearly, $M(G)$ can be completely recovered from $\{0,1,2, \ldots, n\}$ and $q_{G}$, using the fact that $(y, a, h)$ is winning iff $y \geq q_{G}(a)$, so that every quota function $q_{G}$ corresponds to a unique anonymous $(3,2)$ voting rule. Conversely, any quota function can induce a symmetric $(3,2)$ voting rule, as follows: 
Definition 4.2. Given any positive integer $n$ together with any function $q$ having domain equal to some subset of $\{0,1,2, \ldots, n\}$ and codomain equal to $\{0,1,2, \ldots, n\}$ the induced anonymous $(3,2)$ voting rule $G_{q}=\left(n, V_{q}\right)$ is defined by $V_{q}(y, a, h)=1$ iff $q(a)$ is defined with $y \leq q(a)$.

However, $G_{a}$ might not be a $(3,2)$ voting rule, because it might not be monotonic. The somewhat technical conditions on the quota function $q$, necessary and sufficient for $G_{q}$ to be monotonic, are stated in the next theorem, but can be motivated by the example in Fig. 1. Speaking loosely, the graph of a must look somewhat similar to that in Fig. 1: monotonicity requires that it be a step function, that the graph never steps up, that whenever the graph steps down, it only steps down by one unit, and that if the graph "runs off the edge" by crossing the hypotenuse of the triangular grid of points, then it becomes undefined, and it remains undefined for all larger values of $a$ - that is, if the number $a$ of abstentions is so high that the bill fails to pass even when each nonabstainer votes "yes," then any number of abstentions greater than a must also preclude passage. For any fixed number $n$ of voters, the anonymous $(3,2)$ voting rules are then in bijective correspondence with the quota functions $q$ that meet these restrictions. This result is the desired generalization of May's Theorem, and the detailed proof that follows amounts to a check that these four conditions are necessary and sufficient for monotonicity.

Theorem 4.1 (May's Theorem with Abstentions, Version I).

Part A. Let $G=(n, V)$ be any (monotonic) anonymous $(3,2)$ voting rule. Then $G=G_{q}$ for $a$ unique function q satisfying the four conditions below:

(i) The domain Dom(q) of $q$ is equal to some initial segment $\{0,1,2, \ldots, j\}$ of $\{0,1,2, \ldots, n\}$.

(ii) For each $a \in \operatorname{Dom}(q), 0 \leq q(a) \leq n-a$.

(iii) For each $a \in \operatorname{Dom}(q)$ with $q(a)<n-a, a+1 \in \operatorname{Dom}(q)$ and $q(a+1)$ has value $q(a)$ or q(a)-1. $\notin \operatorname{Dom}(q)$.

(iv) For each $a \in \operatorname{Dom}(q)$ with $q(a)=n-a$, either $a+1 \in \operatorname{Dom}(q)$ with $q(a+1)=q(a)-1$ or $a+1$

Part B. Conversely, for any positive integer $n$ and any function $q$ satisfying the four conditions of part A, $G_{q}$ is a (monotonic) anonymous $(3,2)$ voting rule for $n$ voters.

Part $C$. For any positive integer $n$ the map sending $q$ to $G_{q}$ is a bijective orrespondence between the set of functions satisfying the four conditions of part $A$ and the set of anonymous $(3,2)$ voting rules for $n$ voters.

Proof. Let $G$ be an anonymous $(3,2)$ voting rule, and $p=(y, a, h)$ be a minimal winning profile of $G$. Fig. 2 shows the cone over $p$ as a hatched region in the quota function diagram. The profiles along the right arm of $p$ (the line extending to $p$ 's right, with slope 0 ) are obtained by shifting no votes to abstentions, while those in the left arm (the line extending to p's upper left, with slope -1) are obtained by shifting abstentions to yes votes. The figure also shows $p^{R I G H T}$, the right incomparable set of all profiles $p^{\prime \prime}=\left(y^{\prime \prime}, a^{\prime \prime}, h^{\prime \prime}\right)$ that are $\delta$ incomparable with $p$ and also satisfy $a^{\prime \prime}>a$.

Now let $p_{1}, p_{2}, \ldots, p_{r}$ enumerate the minimal winning profiles of $G$ in descending lexicographic order, with $p_{i}=\left(y_{i}, a_{i}, h_{i}\right), 1 \leq 1 \leq r$. These profiles are $\delta$-incomparable, so it follows that $a_{1}<a_{2}<\cdots<a_{r}$ ,$y_{1}>y_{2}>\cdots>y_{r}$, and consequently $h_{1}>h_{2}>\cdots>h_{r}$. Thus $p_{j+1} \in p^{R I G H T}$ for each $j=1,2, \ldots, r-1$. An example with $r=5$ appears as Fig. 3. Monotonicity implies that the winning profiles of $G$ are exactly those in the union $\bigcup_{i=1}^{r} \widehat{p}_{i}$ of the cones over the minimal winning profiles.

From Fig. 3 it is clear that when $M(G) \neq \varnothing$, the graph of $G$ 's quota function $q_{G}$, traced from left to right, consists of alternating sections of left and right arms from the cones $\wedge p_{1}, \wedge p_{2}, \ldots$, respectively, with each section having at least two points, and with the right arm of $\wedge p_{j}$ and the left arm of $\wedge p_{j+1}$ sharing point $\left(y_{j}, a_{j}+h_{j}-h_{j+1}, h_{j+1}\right)$. There are four exceptional situations (the first three of which are not exclusive): 


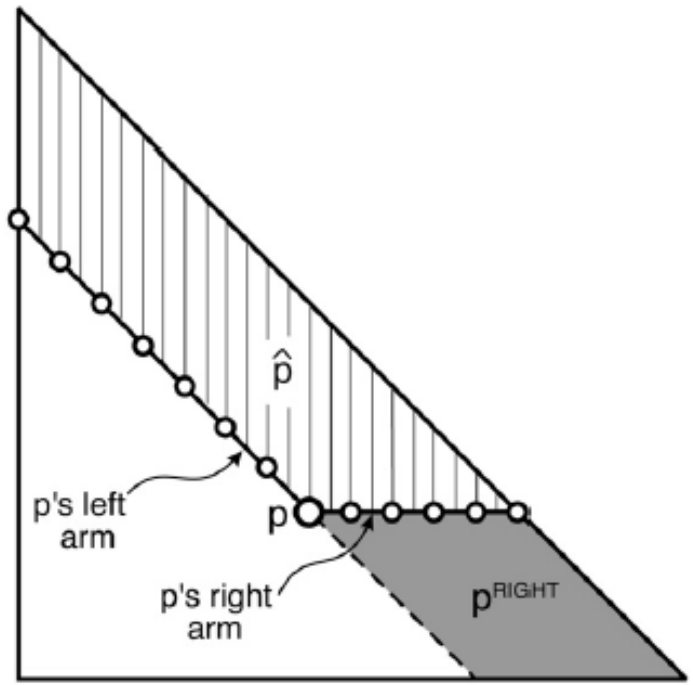

Fig. 2. The cone over $p$ and the right incomparable set of $p$.

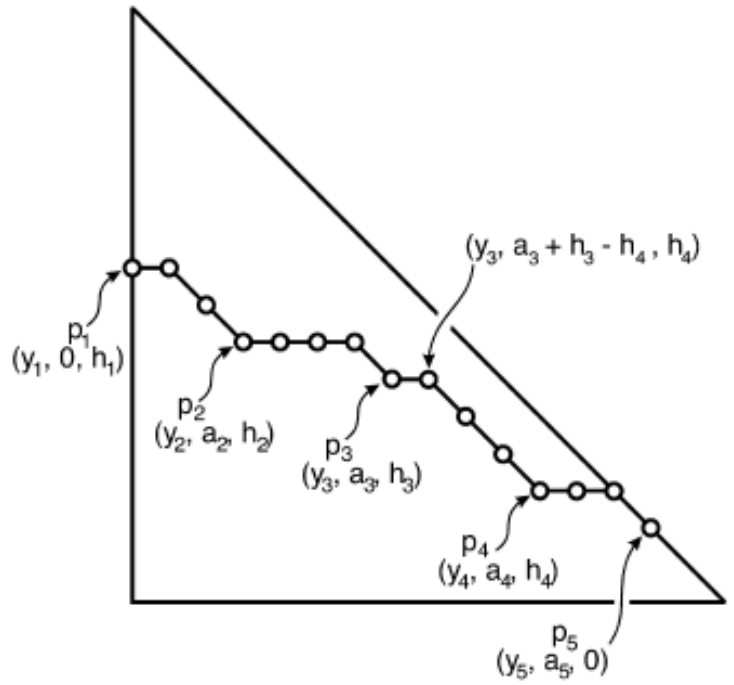

Fig. 3. The graph of $q_{G}$ as alternating left and right arms,

- if $a_{1}=0$ then the left arm of $\widehat{p}_{1}$ is not part of the graph (as happens in Fig. 3 ),

- if $h_{\mathrm{r}}=0$ then the right arm of $\hat{p}_{\mathrm{r}}$ is not part of the graph (as happens in Fig. 3),

- if $\mathcal{M}(G)=\left(\begin{array}{lll}n & 0 & 0\end{array}\right)$ then the graph of $q_{G}$ consists of the single point $(n, 0,0)$, and

- if $A A(C)-\varnothing a_{-}$hac an amntu domain and aranh

This paragraph completely describes $q_{G}=q\left(p_{1}, p_{2}, \ldots, p_{r}\right)$ in terms of $M(G)$. Conditions $A(i)-A$ (iv) follow immediately from this description. (If there are no minimal winning coalitions then the quota function has an empty domain, and satisfies the four conditions vacuously.)

For part B, assume that $q$ is a function satisfying $A(i)-A$ (iv). If $q$ 's graph contains at least two points, then it can be uniquely decomposed into alternating sections of slope 0 and slope -1 , with each section containing at least two points, and each two successive sections having one common point. Use $M(q)$ to denote the set of all leftmost points of sections having slope 0 together with all rightmost points of sections having slope -1 . Then $M(q)$ is a matrix of $\delta$-incomparable profiles, so that the corresponding voting rule $G_{M(q)}$ is an anonymous $(3,2)$ voting rule, and $G_{q}=G_{M(q)}$. If $q$ 's graph contains a unique point it is because $q$ consists of the single point $(n, 0,0)$ so that $M(q)=(n 00)$. If $q$ 's graph does not contain any point, then $q$ has an empty domain, so that $M(q)=\varnothing$. In both situations the voting rule $G_{M(q)}$ is an anonymous $(3,2)$ voting rule, and $G_{q}=G_{M(q)}$.

Part $C$ is straightforward, and is left for the reader.

To illustrate Theorem 4.1 consider the case of 3 voters. Table 2 pairs the 16 allowable quota functions with the corresponding sets of minimal winning profiles.

\subsection{Examples, revisited yet again}

As the number $n$ of voters grows large, we might imagine that the white and black nodes of a quota function diagram, corresponding to the winning and losing anonymous profiles, begin to look more and more like a continuous division of the triangle into lighter and darker regions. We will refer to a diagram showing only these regions, without the individual nodes, as a population generic quota function diagram, or p.g. diagram for short. Each p.g. diagram corresponds to a sequence of anonymous $(3,2)$ voting rules, with one rule for each positive integer $n$. Fig. 4 provides p.g. diagrams for a number of the examples from Section 2.

In Zwicker (2009) we show that one of the conditions equivalent to weightedness for anonymous (3, 2) voting rules is that the winning nodes be separable from the losing ones via a (single) straight line. In particular, Fig. 4 shows that the p.g. diagram for the Hungarian referendum with majority threshold, Example 2.5, is not linearly separable, and so it follows that this example is not weighted.

One consequence of Theorem 4.1 is that for weighted games, monotonicity places limits on the possible slopes of such a line. For p.g. diagrams of weighted games it is not difficult to see that the 
greatest possible slope of a separating line is 0 , as achieved in the case of Absolute Majority Rule. The least possible slope of a separating line is -1 (not pictured). The corresponding voting systems are those requiring a minimum, fixed number of "no" votes to defeat the proposal.

Table 2

Matrices and quota functions for $n=3$ and $j=3$.

\begin{tabular}{|c|c|c|c|c|}
\hline \multirow{2}{*}{$\begin{array}{l}\text { Matrix } \\
\mathcal{M} \\
\end{array}$} & \multicolumn{4}{|c|}{ Quota function } \\
\hline & $\overline{q(0)}$ & $q(1)$ & $q(2)$ & $q(3)$ \\
\hline $\bar{\theta}$ & - & - & - & - \\
\hline$\left(\begin{array}{lll}3 & 0 & 0\end{array}\right)$ & 3 & - & - & - \\
\hline$\left(\begin{array}{lll}2 & 1 & 0\end{array}\right)$ & 3 & 2 & - & - \\
\hline$\left(\begin{array}{lll}2 & 0 & 1\end{array}\right)$ & 2 & 2 & - & - \\
\hline$\left(\begin{array}{lll}1 & 2 & 0\end{array}\right)$ & 3 & 2 & 1 & - \\
\hline$\left(\begin{array}{lll}1 & 1 & 1\end{array}\right)$ & 2 & 1 & 1 & - \\
\hline$\left(\begin{array}{lll}1 & 0 & 2\end{array}\right)$ & 1 & 1 & 1 & - \\
\hline$\left(\begin{array}{lll}0 & 3 & 0\end{array}\right)$ & 3 & 2 & 1 & 0 \\
\hline$\left(\begin{array}{lll}0 & 2 & 1\end{array}\right)$ & 2 & 1 & 0 & 0 \\
\hline$\left(\begin{array}{lll}0 & 1 & 2\end{array}\right)$ & 1 & 0 & 0 & 0 \\
\hline$\left(\begin{array}{lll}0 & 0 & 3\end{array}\right)$ & 0 & 0 & 0 & 0 \\
\hline$\left(\begin{array}{lll}2 & 0 & 1 \\
1 & 2 & 0\end{array}\right)$ & 2 & 2 & 1 & - \\
\hline$\left(\begin{array}{lll}2 & 0 & 1 \\
0 & 3 & 0\end{array}\right)$ & 2 & 2 & 1 & 0 \\
\hline$\left(\begin{array}{lll}1 & 1 & 1 \\
0 & 3 & 0\end{array}\right)$ & 2 & 1 & 1 & 0 \\
\hline$\left(\begin{array}{lll}1 & 0 & 2 \\
0 & 3 & 0\end{array}\right)$ & 1 & 1 & 1 & 0 \\
\hline$\left(\begin{array}{lll}1 & 0 & 2 \\
0 & 2 & 1\end{array}\right)$ & 1 & 1 & 0 & 0 \\
\hline
\end{tabular}

a)

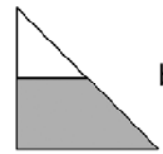

b)

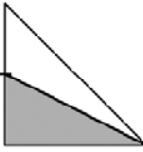

c)

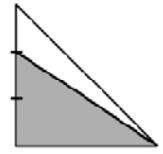

d)
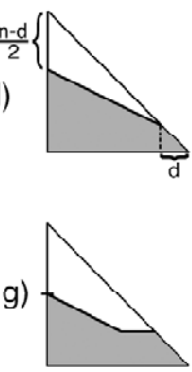

e)

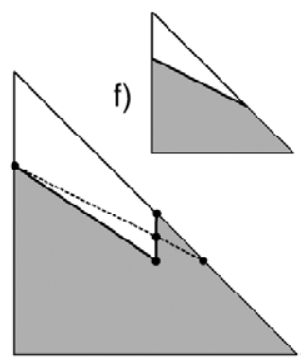

Fig. 4. Population generic quota function diagrams for: (a) absolute majority rule, (b) simple majority rule, (c) ratio quota with $r=2 / 3$, (d) difference quota, (e) the rule for ratifying treaties in the U.S. Senate, (f) the "soft quorum" system, Example 2.6, (g) the Hungarian referendum, with majority threshold of 1/4.

The slope of the separating line for simple majority rule is $-1 / 2$, and this line passes through the right corner $(n, 0,0)$ of the triangle, Fig. 4b. The other p.g. diagrams whose separating line passes through this corner correspond exactly to the ratio quota systems, while the diagrams whose separating lines have slopes of $-1 / 2$ likewise correspond to the difference quota systems. (The distances marked $(n-d) / 2$ and $d$ in Fig. $4 d$ are based on the assumption that each leg of the triangle has length $n$.)

Our soft quorum example in Fig. $4 \mathrm{f}$ is a difference weighted system. However, this is something of an accident; closely related examples, that similarly have something of the effect of a quorum because their separating lines cross the hypotenuse, are neither difference nor ratio weighted. Loosely speaking, these may be thought of as interpolants between absolute and simple majority rules, or mixes of ratio and difference quotas. It is worth mentioning that naive definitions of "weighted voting with abstention" tend to reduce, in the anonymous case, either to ratio quota or to difference quota systems. Thus, the greater generality of the definition we employ is necessary if, for example, one wishes to classify soft quorum systems as weighted voting. 
Absolute and simple majority rules now appear to be two extremes within a larger range of natural possibilities, which encompass such decision rules as the soft quorum example we discuss. It would be interesting to compare the members of this family in the same spirit as the comparison of simple and majority rules appearing in Dougherty and Edward (forthcoming).

\begin{tabular}{|c|c|c|c|c|c|c|c|c|}
\hline$\downarrow j \| \rightarrow n$ & 2 & 3 & 4 & 5 & 6 & 7 & 8 & 9 \\
\hline 2 & 4 & 5 & 6 & 7 & 8 & 9 & 10 & 11 \\
\hline 3 & 8 & 16 & 32 & 64 & 128 & 256 & 512 & 1024 \\
\hline 4 & 16 & 66 & 352 & 2431 & 21760 & 252586 & 3803648 & - \\
\hline 5 & 32 & 352 & 9304 & 683464 & 161960220 & - & - & - \\
\hline 6 & 64 & 2431 & 683464 & - & - & - & - & - \\
\hline 7 & 128 & 21760 & 161960220 & - & - & - & - & - \\
\hline 8 & 256 & 252586 & - & - & - & - & - & - \\
\hline 9 & 512 & 3803648 & - & - & - & - & - & - \\
\hline 10 & 1024 & - & - & - & - & - & - & - \\
\hline
\end{tabular}

The sharp jag or discontinuity in the separating boundary for the Senate Treaty Ratification diagram, Fig. $4 \mathrm{e}$, reflects the nonmonotonicity induced by the "hard" quorum built in to the voting rules. It is directly related to the sort of pathology discussed in footnote 3 . The dashed line we have added to this figure is the separating line from the soft quorum system.

Our purpose in comparing these two systems is not to actually suggest that the soft quorum system should replace the current one for treaty ratification. Important differences remain, and the pathology we have mentioned is hardly extreme. Rather our purpose in comparing these two systems is to point out that there are some interesting alternatives to the voting systems we currently employ. These alternatives are not very complicated, but they do not seem to have been considered heretofore. They have some attractive properties that might make them worth considering for some future real application. We expand on these comments in the final section of the paper.

\section{Counting}

We may deduce two counting facts from the previous work. The first concerns the number of anonymous voting rules with 3 levels of approval, and is derived from Theorem 4.1. The second exhibits a certain surprising symmetry for anonymous voting rules, and follows from the material in Section 3. We were led to these results by Table 3 , which provides the number of anonymous $(j, 2)$ voting rules for small values of $j$ and $n$.

\subsection{Yes-no voting with abstention: there are $2^{n+1}$ anonymous rules}

Theorem 5.1. The number of anonymous $(3,2)$ voting rules with $n$ players is $2^{n+1}$.

Proof. (i) Temporarily fix some integer $a$ with $0 \leq a \leq n-1$ and consider those step functions satisfying $q(a)=n-a$ and $\operatorname{Dom}(a)=\{0,1, \ldots, a\}$. For each integer $b$ with $0 \leq b \leq a-1$ we can freely choose $q(b)-q(b+1)$ to have a value of 0 or of 1 . Each such a sequence of choices yields a "good" quota function - one satisfying the Theorem 4.1 conditions. Hence, there are $2^{a}$ good quota functions having the stated domain. As a ranges from 0 to $n$ we get $2^{0}+\cdots+2^{n}=2^{n+1}-1$ good quota functions. Finally, we get the stated result by adding the single quota function with empty domain.

\subsection{A form of duality}

Theorem 5.2. The number of anonymous $(j, 2)$ voting rules with $n$ voters $(j \geq 2, n \geq 2)$ coincides with the number of anonymous $(n+1,2)$ voting rules with $j-1$ voters.

Proof. Let $A G_{j, k, n}$ denote the set of all anonymous $(j, k)$ voting rules for $n$ voters. 
We construct a bijection $\Pi_{j, n}: P(n, j) \rightarrow P(j-1, n+1)$, described below. The desired bijection $\Pi_{j, 2, n}$ : $A G_{j, 2, n} \rightarrow A G_{n+1,2, j-1}$, is defined from $\pi_{j, n}$ (henceforth, $\pi$ ) by

$$
(n, \vee) \rightarrow\left(j-1, \vee \circ \pi^{-1}\right)
$$

That $\Pi$ takes $A G_{j, 2, n}$ bijectively to $A G_{n+1,2, j-1}$ follows immediately from the fact, proved below, that $\Pi$ preserves the $\delta$ ordering: whenever $a \delta b, \pi(a) \delta \pi(b)$.

Note that this $\delta$ preservation property also implies that

(a) From the set $M$ associated with a rule $G$ we may easily calculate the set for $\Pi(G)$; simply apply $\pi$ separately to the individual members of $M$; and that

(b) The same rule $(n, V) \rightarrow\left(j-1, \vee \circ \Pi^{-1}\right)$ yields a similar bijection $\Pi_{j, k, n}: A G_{j, k, n} \rightarrow A G_{n+1, k, j-1}$ for any integer $k>2$; hence for each $k$ the number of anonymous voting systems for $n$ voters with $j$ levels of input approval and $k$ levels of output approval is equal to the number of such systems for $j-1$ voters with $n+1$ levels of input approval and $k$ levels of output approval.

To facilitate the later argument, we describe $\pi$ in the form of a step-by-step algorithm for calculating outputs from inputs:

[Start with a $j$-tuple whose entries sum to $n$. Example: $(0,3,1,0,2)$ is in $P(6,5)$.]

Step 1: Delete all zeros, leaving all commas in place.

[Now there are $j-1$ commas, and the integers sum to $n$. Example: $(, 3,1,2)$.]

Step 2: Replace each integer a $j$ with a string of $j$ asterisks.

[Now there are $j-1$ commas and $n$ asterisks. Example: $\left(,{ }^{* * *}, *,, * *\right)$.]

Step 3: Reverse the string from left to right, while simultaneously switching commas to asterisks and asterisks to commas.

[Now there are $n$ commas and $j-1$ asterisks. Example: $\left(,,^{* *}, *,,{ }^{*}\right)$.]

Step 4: Replace each maximal-length string of successive asterisks with the length of the string.

[Now there are $n$ commas and the integers sum to $j-1$. Example: $(, 2,1,1,1)$.]

Step 5: Insert zeros where needed to create a syntactically legal tuple.

[Now we have an $n+1$ tuple whose entries sum to $j-1$. Example: $(0,0,2,1,0,0,1)$.]

From the comments in square brackets, it is apparent that an input in $P(n, j)$ yields an output in $P(j-$ $1, n+1)$. Notice that if we write new instructions for reversing each of the five steps, and performing them in reverse order (starting by undoing Step 5), the results are the same as the original instructions. Thus $\pi$ is its own inverse (more precisely, $\pi^{-1}{ }_{j, n}=\pi_{n+1}, j-1$ ), and $\pi$ is bijective.

Claim. If $a^{\prime}$ is obtained from a by a left shift by one space of a single 1 (for example, $(0,3,1,1,1)$ is obtained from $(0,3,1,0,2)$ by shifting one of the two $1 \mathrm{~s}$ in the fifth place into the fourth place), then $\Pi\left(a^{\prime}\right)$ is similarly obtained from $\pi(a)$ by such a left shift.

From the claim, it follows immediately that $\pi$ preserves $\delta$, as $c \delta a$ holds exactly when $c$ may be obtained from a through a sequence of such shifts.

Proof of Claim. We trace the effect of a single left shift through the five steps of $\pi$. After Step 2, the symbol string for $a^{\prime}$ is obtained from that for $a$ by transposing some adjacent pair ,* of symbols (so they become ${ }^{*}$,). After the double reversal of Step 3, the net effect is the same - some two successive symbols, ${ }^{*}$ become ${ }^{*}$, From this it is clear that $\Pi\left(a^{\prime}\right)$ is obtained from $\Pi(a)$ by a single left shift.

An immediate consequence of the two previous theorems is that the number of anonymous voting rules with $j$ input levels and $n=2$ voters is $2^{j+1}$. 
Anonymous simple games, corresponding to anonymous $(2,2)$ voting rules, are simple indeed. We have shown, however, that when $j=3$ the number of such rules grows exponentially in the number $n$ of voters; we note that for even larger $j$ values, the growth rate appears to be greater yet. More generally, the extension of anonymous simple games to three or more levels of input approval provide a wide collection of voting rules with a considerable level of complexity. For instance, not every such rule is weighted, and so it would be convenient to get specific characterizations of weightedness for them. (This has been done for the $(3,2)$ case only, in Zwicker, 2009.)

\section{Classifying anonymous ( $\mathrm{j}, \mathrm{k})$ nules}

A matricial presentation, $M(G)$, for anonymous ( j, 2) voting rules has been given in Section 3 . We outline here how to extend the matricial classification method to the context of $k>2$ output levels. We refer the reader to Freixas (2005a, 2005b), Freixas and Zwicker (2003) for examples within this category. Our principal concern here is with the case $k>2$, for which some specialized terminology is appropriate.

Definition 6.1. Let $G=(n, \vee)$ be an anonymous $(j, k)$ voting rule for $n$ voters, and $p \in P(n, j)$. If $\vee(p)=i$, we say that $p$ is an $i$-winning profile. If $p$ is an $i$-winning profile and if for every profile $p^{\prime} \in P(n, j)$ with $p \delta p^{\prime}, p^{\prime}$ is an $r$-winning profile with $r<i$, then $p$ is an $i$-minimal winning profile. We will use $M^{i}(G)$ to denote $\{p \in P(n, j) \mid p$ is an $i$-minimal winning profile of $G\}$, so that the cone over $p, \wedge p=\{p \in P(n, j) \mid$ $q \delta p$ or $q=p\}$, contains (by monotonicity) only $r$-winning profiles for $r \geq i$.

Given the sets $M^{i}(G)$, for $1 \leq i \leq k-1$, of $i$-minimal winning profiles for an anonymous $(j, k)$ voting rule $G$ $=(n, V)$, we can easily recover the value function $V$ for $G$. The set $M^{i}(G)$ is empty only if $V(p) \neq i$ for all profiles $p$. It is useful to present $M^{i}(G)$ in the form of a matrix, $M^{i}(G)$, in which each row enumerates a different profile $p=\left(1 p, 2 p, \ldots, j p\right.$ in $M^{i}(G)$, that is $i$-minimal winning for $G$, and the rows are listed in descending lexicographic order. Thus each anonymous $(j, k)$ voting rule $G$ for $n$ players yields a unique sequence of $k-1$ matrices $\left[M^{1}, \ldots, M^{k-1}\right](G)$ having five properties, including four internal properties for each nonempty matrix $M^{i}$ :

(1) each row has length $j$,

(2) each row has sum $n$,

(3) the rows are distinct and incomparable in the $\delta$ order,

(4) the rows are listed in descending lexicographic order;

as well as one additional external property:

(5) $q \not \subset \wedge p$ whenever $p \in M^{i}, q \in M^{h}$ with $1 \leq h<i \leq k-1$.

Conversely, it is easy to see that each sequence of matrices $M^{1}, \ldots, M^{k-1}$ with these five properties enumerates $\left[M^{1}, \ldots, M^{k-1}\right](G)$ for a unique anonymous $(j, k)$ voting rule $G$ for $n$ players.

For instance, suppose that $j=3, k=3$ and $n=9$ so that we need an $M^{1}$ and an $M^{2}$. Let $M^{2}=\left(\begin{array}{ll}6 & 0\end{array}\right)$ and $\mathcal{M}^{1}=\left(\begin{array}{lll}5 & 0 & 4 \\ 4 & 3 & 2\end{array}\right)$. They define an anonymous $(3,3)$ voting rule in which:

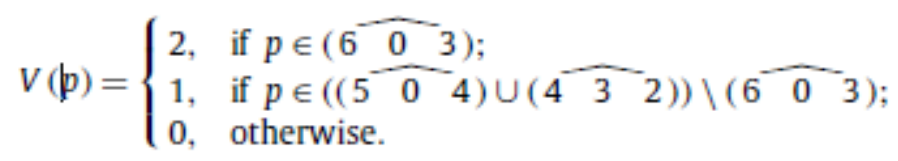

Consequently, given any $j, k$ and $n$ and we can generate and count all anonymous $(j, k)$ voting rules for $n$ players. As an example, if $(j, k, n)=(2,3,2)$ we obtain 10 length-2 sequences of matrices which give rise to 10 anonymous $(2,3)$ voting rules for 2 players, 20 length-3 sequences of matrices which give rise to 20 anonymous $(2,4)$ voting rules for 2 players, 33 length-2 sequences of matrices if $(j, k, n)$ $=(3,3,2)$, and so on. 


\section{Conc luding remarks}

One model for writing a paper in social choice is to begin with a list of desirable properties for a certain decision-making procedure, and to conclude by describing the (possibly empty) class of procedures that meet these criteria. But in real life, we do not always know what we are looking for until we sample the range of available options. (Shopping for a new piece of furniture is one example that comes to mind here.) In particular, there may be candidate properties for our list, whose existence we do not suspect until we see them in action, in some example.

Our goal has been to describe the range of options available for anonymous voting with multiple levels of approval in the input. While our model fits many real world decision-making procedures, it seems as if choices of particular procedures have historically been made without an appreciation of this full range. The range is richer than may have been suspected, as witnessed by the exponential growth rate in the number of procedures, and in significant contrast to the situation for anonymous voting with only two levels of input approval. Yet it is not so rich that it cannot be parsed. This is particularly so if one limits oneself to the rather natural subclass of weighted procedures, as is shown in Zwicker (2009).

\subsection{A final note on the real world}

Much of the current interest in abstention arose from the observation that the simple game model is, in fact, a poor one in terms of real voting. Without a doubt, the models discussed here, and in a number of the references, move us much closer to real voting practice. But it would be irresponsible to leave the impression that these models, or any others, could ever fully reflect reality. For one thing, legislatures tend to evolve sets of working practices that are too complex and informal to be described mathematically, and that may not even be written down. In this connection, see a number of comments in Freixas and Zwicker (2003).

Even more misleading is that legislators do not necessarily follow their own rules, and the official record may not fully reflect this fact. An extreme example is provided in Marcy (1953), which begins

On June 13, 1952, with two senators on the floor, the Senate of the United States gave its advice and consent to the ratification of three treaties which thereby become a part of the supreme law of the land. One of the senators did not vote. The other voiced his "aye" while serving as presiding officer.

It is clear from the article that we would not know the full story were it not for personal testimony of some witnesses on the Senate floor that day. The entire article would make interesting reading, and sound an appropriately cautionary note, to anyone who might be using published rules as the sole basis of their understanding of how some voting system works in practice.

\section{Acknowledgments}

The authors wish to thank J. Dibbell, R. Ramón and two anonymous referees whose comments significantly improved the exposition of the paper.

\section{References}

Amer, R., Carreras, F., Magaña, A., 1998. The Banzhaf-Coleman index for games with $r$ alternatives. Optimization 44, 175-198.

Axtman K., 2003. As redisdistricting tale ends, fight echoes beyond Texas. The Christian Science Monitor 19, 2. Newspaper article in the September 19 edition.

Bilbao, J.M., 2000. Cooperative Games on Combinatorial Structures. Theory and Decision Library, Series C. Kluwer Academic Publishers, Dordrecht.

Bilbao, J.M., Fernández, J.R., Jiménez, N., López, J.J., 2008a. Biprobabilistic values for bicooperative games. Discrete Appl. Math. 156, 2698-2711. 
Bilbao, J.M., Fernández, J.R., Jiménez, N., López, J.J., 2008b. The Shapley value for bicooperative games. Ann. Oper. Res. 158, 99-115.

Bolger, E.M., 1986. Power indices for multicandidate voting games. Int. J. Game Theory 15, 175-186.

Bolger, E.M., 1993. A value for games with $n$ players and $r$ alternatives. Int. J. Game Theory 22, 319334.

Bolger, E.M., 2000. A consistent value for games with $n$ players and $r$ alternatives. Int. J. Game Theory 29, 93-99.

Côrte-Real, P.P., Pereira, P.T., 2004. The voter who wasn't there: Referenda, representation and abstention. Soc. Choice Welf. 22, 349-369.

Dougherty, K., Edward, J., forthcoming. The properties of simple vs. absolute majority rule: Cases where absences and abstentions are important. J. Theor.Politics.

Felsenthal, D.S., Machover, M., 1997. Ternary voting games. Int. J. Game Theory 26, 335-351.

Felsenthal, D.S., Machover, M., 1998. The Measurement of Voting Power: Theory and Practice, Problems and Paradoxes. Edward Elgar, Cheltenham.

Fishburn, P.C., 1973. The Theory of Social Choice. Princeton University Press, Princeton.

Freixas, J., 2005a. Banzhaf measures for games with several levels of approval in the input and output. Ann. Oper. Res. 137, 45-66.

Freixas, J., 2005b. The Shapley-Shubik power index for games with several levels of approval in the input and output. Decis. Support Syst. 39, 185-195.

Freixas, J., Zwicker, W.S., 2003. Weighted voting, abstention, and multiple levels of approval. Soc. Choice Welf. 21, 399-431.

Hsiao, C.R., Raghavan, T.E.S., 1993. Shapley value for multichoice cooperative games I. Games Econ. Behav. 5, 240-256.

Lindner I., 2005. Voting power in games with abstentions: A probabilistic characterization and a special case of Penrose's Limit theorem. Technical report, forthcoming as a CORE discussion paper. Available at SSRN: http://ssrn.com/abstract=885909.

Marcy, C., 1953. A note on treaty ratification. Amer. Polit. Sci. Rev. 47, 1130-1133.

May, K., 1952. A set of independent, necessary and sufficient conditions for simple majority decision. Econometrica 20, 680-684.

Rubenstein, A., 1980. Stability of decision systems under majority rule. J. Econ. Theory 23, 150-159.

Taylor, A.D., Pacelli, A., 2008. Mathematics and Politics, second ed. Springer Verlag, New York.

Taylor, A.D., Zwicker, W.S., 1992. A characterization of weighted voting. Proc. Amer. Math. Soc. 115 , 1089-1094.

Taylor, A.D., Zwicker, W.S., 1999. Simple Games: Desirability Relations, Trading, and Pseudoweightings. Princeton University Press, Princeton, NJ.

Tchantcho, B., Diffo Lambo, L., Pongou, R., Engoulou, B.M., 2008. Voters' power in voting games with abstention: Influence relation and ordinal equivalence of power theories. Games Econ. Behav. 64, 335-350.

Uleri, P.V., 2002. On referendum voting in Italy: YES, NO, or non-vote? How Italian parties learned to control referendums. Europ. J. Polit. Res. 41, 863-883.

Zwicker, W.S., 2009. Anonymous voting rules with abstention: Weighted voting. In: Brams, S.J., Gehrlein, W.V., Roberts, F.S. (Eds.), The Mathematics of Preference, Choice, and Order: Essays in Honor of Peter C. Fishburn. Springer, Heidelberg, pp. 239-258. 